\title{
Peak-to-Average Power Ratio Reduction Method Based on Partial Transmit Sequence and Discrete Fourier Transform Spreading
}

\author{
Nahla Al Harthi, Zhongfeng Zhang, Daejin Kim and Seungwon Choi *
}

Citation: Al Harthi, N.; Zhang, Z.; Kim, D.; Choi, S. Peak-to-Average Power Ratio Reduction Method Based on Partial Transmit Sequence and Discrete Fourier Transform Spreading. Electronics 2021, 10, 642 https://doi.org/10.3390/electronics 10060642

Academic Editor: Leonardo Pantoli

Received: 10 February 2021

Accepted: 8 March 2021

Published: 10 March 2021

Publisher's Note: MDPI stays neutral with regard to jurisdictional claims in published maps and institutional affiliations.

Copyright: (c) 2021 by the authors. Licensee MDPI, Basel, Switzerland. This article is an open access article distributed under the terms and conditions of the Creative Commons Attribution (CC BY) license (https:// creativecommons.org/licenses/by/ $4.0 /)$.
Department of Electronic and Computer Engineering, Hanyang University, Wangsimni-ro 222, Seongdong-gu, Seoul 04763, Korea; nahla.harthi@dsplab.hanyang.ac.kr (N.A.H.); zhongfeng.zhang@dsplab.hanyang.ac.kr (Z.Z.); kdj317@dsplab.hanyang.ac.kr (D.K.)

* Correspondence: choi@dsplab.hanyang.ac.kr

\begin{abstract}
Recently, filter bank multicarrier with offset quadrature amplitude modulation (FBMC/ OQAM) has received increasing attention from researchers, owing to its merits and superior spectral efficiency. High peak-to-average power ratio (PAPR) occurs in approximately all multicarrier systems, including FBMC/OQAM, and may cause bit-error-rate (BER) degradation if not appropriately handled. Conventional PAPR reduction methods for orthogonal frequency division multiplexing (OFDM), such as partial transmit sequence (PTS), selective mapping (SLM), and discrete Fourier transform (DFT) spreading, are ineffective in FBMC/OQAM because of the different structure of the symbols. This study proposes a novel method combining DFT spreading and PTS methods to reduce the PAPR of FBMC/OQAM systems with reasonable computational complexity. Numerical results obtained from various computer simulations show that the proposed method achieves a noticeable enhancement in the PAPR performance of the FBMC/OQAM signal compared to other existing methods without affecting the BER performance. Further, the computational complexity analysis and BER performance of the proposed method are presented in comparison to typical existing methods. From our computer simulations, the proposed method reduces the PAPR by approximately $32.8 \%$ compared to that of the conventional methods, and the BER performance is improved by $25 \%$ with a high-power amplifier effect.
\end{abstract}

Keywords: filter bank multicarrier with offset quadrature amplitude modulation; orthogonal frequency division multiplexing; peak-to-average power ratio; discrete Fourier transform; partial transmit sequence; bit-error-rate

\section{Introduction}

Filter bank multicarrier with offset quadrature amplitude modulation (FBMC/OQAM) has attracted attention as a strong competitor of orthogonal frequency division multiplexing (OFDM) in 5G technologies [1]. Although OFDM systems are very efficient, their spectral efficiency deteriorates with the addition of a cyclic prefix (CP) and a high out-of-band emission caused by rectangular filtering [2]. However, FBMC/OQAM achieves better side-lobe suppression than some existing suppression methods in OFDM signals, with a moderate increase in complexity [3]. OFDM requires perfect carrier synchronization between different users to avoid interference in the uplink. However, FBMC separates different signal frequencies using pulse-shaping filters, which makes time synchronization unnecessary [1,2]. Although FBMC has attractive merits, it also experiences a high peakto-average power ratio (PAPR) caused by the inverse discrete Fourier transform (IDFT) at the transmitter.

Conventional PAPR reduction schemes, such as discrete Fourier transform (DFT) spreading, clipping and filtering, partial transmit sequence (PTS), tone reservation (TR), and selective mapping (SLM), effectively reduce the PAPR but at the expense of high computational complexity, degraded spectral efficiency, and an increase in bit-error rate (BER) [4]. 
Conventional methods reduce the peaks of each symbol block separately; however, in FBMC/OQAM, the symbol blocks overlap. Consequently, the PAPR reduction is marginal compared to OFDM. The authors in [5] proposed a tone reservation technique with sliding windows, where the sliding windows are overlapped to prevent out-of-window peak regrowth. PTS has been intensively studied [6-11]; the authors in [6] combined PTS and TR techniques to reduce the peaks of FBMC signal segments. In [11], the authors proposed a method based on PTS, referred to as multi-block joint optimization with a dynamic programming algorithm.

DFT spreading has gained popularity as a very efficient PAPR reduction scheme in OFDM systems, especially for uplink applications that require less power consumption. Unfortunately, this method does not achieve desirable results in FBMC signals. Earlier studies focused on exploiting the DFT spreading effect to improve PAPR reduction in FBMC by employing diverse techniques such as the identically time-shifted multicarrier (ITSM) condition, generalized DFT spreading (GDFT), pruned DFT, and pre-coding technique [12-14]. In [14], the authors proposed a method based on pruned DFT spreading that effectively reduced the PAPR, sacrificing the system's computational complexity.

In this study, we propose a novel method for reducing PAPR in FBMC/OQAM by combining DFT spreading, satisfying the ITSM condition of the phase factors, and a modified PTS method that complies with the structure of FBMC/OQAM. In the modified PTS method, partitioning and searching for the candidate with the least PAPR are performed at the real and imaginary parts of the FBMC/OQAM, respectively. Furthermore, the overlapping of the previous symbol parts is considered while calculating the candidates' PAPR at each part.

The remainder of this paper is organized as follows. Section 2 introduces the FBMC/OQAM signal structure with a concrete definition of PAPR. In Section 3, the proposed method is explained in detail. Finally, the simulation results and conclusion are presented in Sections 4 and 5, respectively.

\section{FBMC/OQAM Structure}

Figure 1 shows the structure of the FBMC/OQAM transmitter. The FBMC/OQAM signal structure is varied from that of the OFDM by replacing the $\mathrm{CP}$ with a pulse-shaping filter bank and OQAM modulation. Figure 2 shows the FBMC signal structure; it mainly consists of $\mathrm{M}$ overlapped data blocks. Each symbol contains $\mathrm{N}$ sub-carriers, and the $\mathrm{n}^{\text {th }}$ sub-carrier on the $\mathrm{m}^{\text {th }}$ block carries the QAM-modulated data symbols, $\mathrm{x}_{\mathrm{n}, \mathrm{m}}$. The signal in the time domain is expressed as follows [6]:

$$
\begin{gathered}
s(t)=\sum_{n=0}^{N-1} \sum_{m=0}^{M-1} x_{n, m} h(t)_{n, m^{\prime}} 0 \leq t \leq\left(M+K-\frac{1}{2}\right) T \\
s(t)=\sum_{n=0}^{N-1} \sum_{m=0}^{M-1}\left\{c_{n, m} h(t-m T)+j d_{n, m} h\left(t-m T-\frac{T}{2}\right)\right\} e^{j \varphi_{n, m}}
\end{gathered}
$$




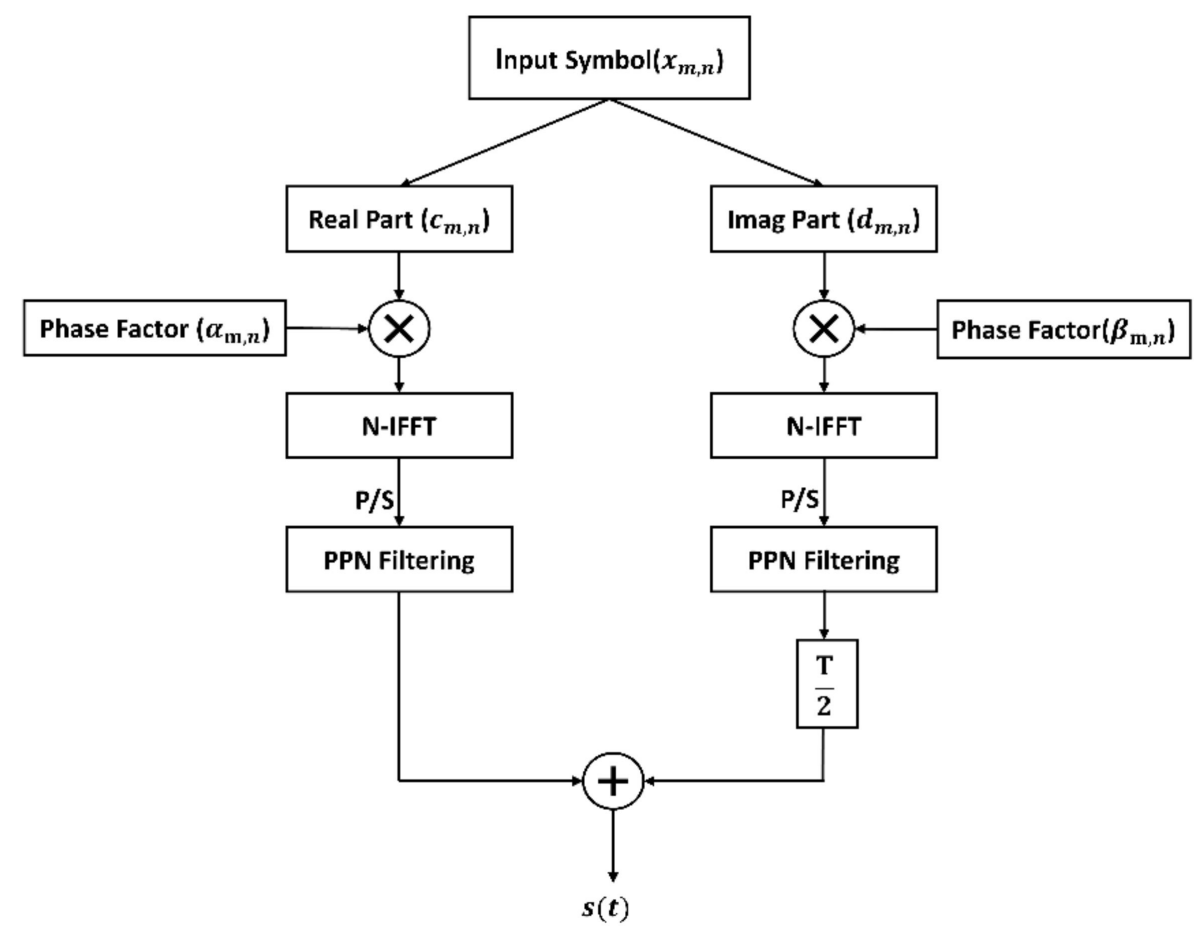

Figure 1. Transmitter structure of FBMC/OQAM (filter bank multicarrier with offset quadrature amplitude modulation).

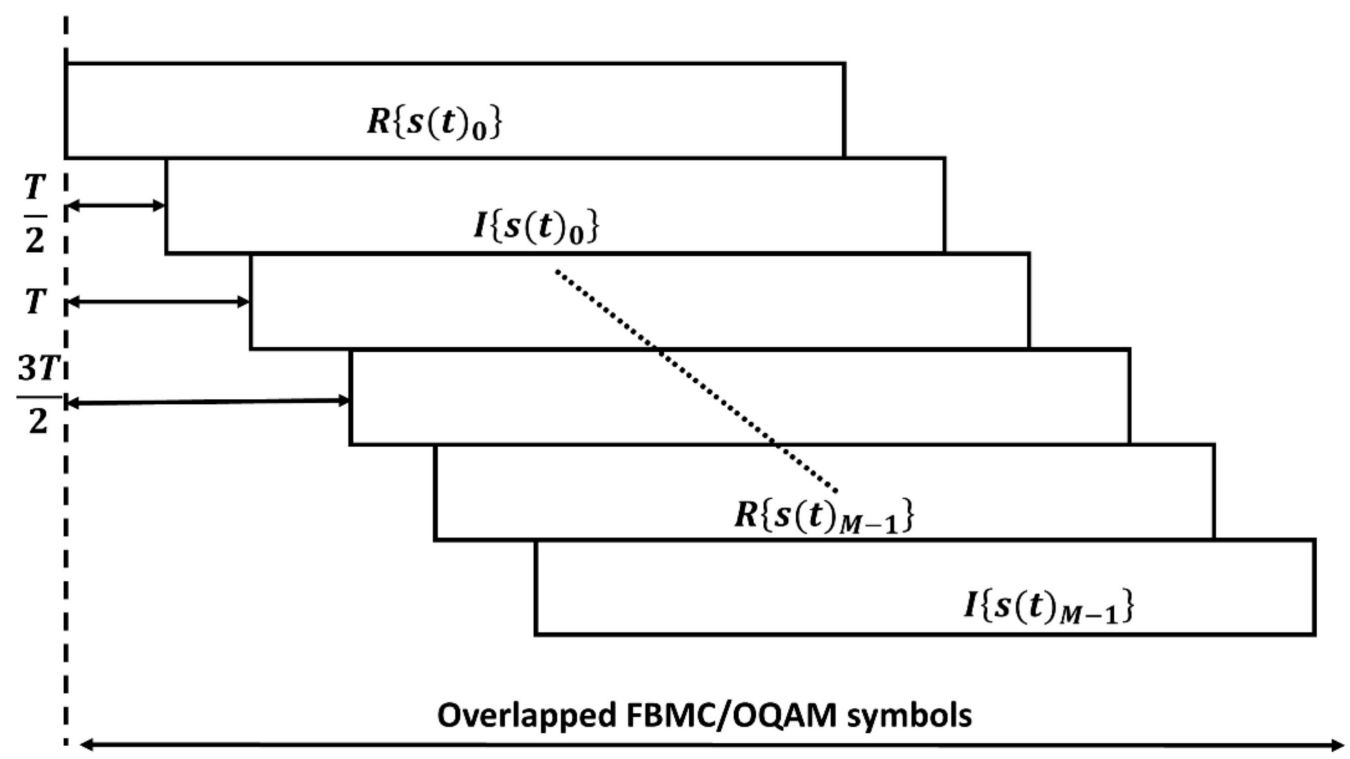

Figure 2. Structure of FBMC/OQAM (filter bank multicarrier with offset quadrature amplitude modulation) signal.

As shown in Equation (2), the complex input symbols $x_{n, m}$ are converted to real symbols consisting of real $\left(c_{n, m}\right)$ and imaginary parts $\left(d_{n, m}\right)$. The resulting symbols alternate between each other by multiplication with phase rotation factors that follow the rules in (3) and (4). The orthogonality between neighboring symbols can be preserved by staggering the imaginary part by half a symbol period. However, this operation increases the data rate to 2 . The previous steps are part of the OQAM pre-processing on the transmitter side.

$$
\alpha_{\mathrm{n}, \mathrm{m}}=\left\{\begin{array}{cc}
1 \text { or }-1 & \mathrm{n}=\text { even } \\
\mathrm{j} \text { or }-\mathrm{j} & \mathrm{n}=\text { odd }
\end{array}\right.
$$




$$
\beta_{n, m}= \begin{cases}j \text { or }-j & n=\text { even } \\ 1 \text { or }-1 & n=\text { odd }\end{cases}
$$

The prototype filter $h(t)$ in (2) is used for pulse shaping each sub-carrier. We adopted the PHYDYAS filter in [15] for the simulations. The length $\mathrm{h}(\mathrm{t})$ is equal to $\mathrm{KT}$, where $\mathrm{K}$ and $\mathrm{T}$ represent the overlapping factor and symbol duration, respectively. Depending on the value of the overlapping factor, the FBMC/OQAM symbol overlaps with a maximum of $(\mathrm{K}-1)$ symbols. The filter's impulse response in the time domain is expressed as follows [16]:

$$
\mathrm{h}(\mathrm{t})=1+2 \sum_{\mathrm{k}=1}^{\mathrm{K}-1} \mathrm{H}_{\mathrm{K}} \cos \left(2 \pi \frac{\mathrm{kt}}{\mathrm{KT}}\right)
$$

The total number of filter coefficients is $2 \mathrm{~K}-1$; the coefficient values for different $\mathrm{K}$ values are presented in Table 1 [16].

Table 1. PHYDYAS filter coefficients.

\begin{tabular}{ccccc}
\hline K & H0 & H1 & H2 & H3 \\
\hline 2 & 1 & 0.707107 & - & - \\
3 & 1 & 0.911438 & 0.411438 & - \\
4 & 1 & 0.971960 & 0.707107 & 0.235147 \\
\hline
\end{tabular}

The FBMC/OQAM system consists of synthesis and analysis filter banks placed at the transmitter and receiver sides, respectively. The synthesis and analysis filters complement each other and satisfy the Nyquist criterion. The PHYDYAS project introduced two different approaches of implementing the filter bank, which are the frequency spreading (FS) and the polyphase network (PPN) [16]. FS-FBMC requires KT point IFFT/FFT because the data are spread in frequency while PPN-FBMC requires only N point IFFT/FFT. For our study, we chose the PPN filter structure as it requires less computational complexity than FS-FBMC. A filter bank based on PPN structure is constructed from $\mathrm{N}$ filters by shifting the prototype filter's response by multiples of $1 / \mathrm{N}$ in the frequency domain. All the filters in the network are linear phased. The filter's length depends on the number of the sub-carriers $\mathrm{N}$ and $\mathrm{K}$; the higher the number of sub-carriers, the more complex the filter design. In the transmitter side, each IFFT block's output is copied K times to create a vector of length KT. This vector is then filtered by the sampled prototype filter, $\mathrm{h}(\mathrm{t})$. After that, the real and imaginary parts are summed by staggering the imaginary part, as shown in Figure 1. Finally, the sequence is multiplied by the phase term $\varphi_{\mathrm{n}, \mathrm{m}}=\mathrm{n}\left(\frac{2 \pi \mathrm{t}}{\mathrm{T}}+\frac{\pi}{2}\right)$ and transmitted.

PAPR determines the ratio of the multicarrier signal's peak power over the average power. As PAPR value increases, it indicates that the system requires high-power amplifiers (HPAs) to operate in their saturation region, which is critical because any further increase in the signal amplitude pushes the HPA to operate in its nonlinear region, eventually causing distortion to the transmitted signal. In OFDM systems, the reduction methods are separately applied over each symbol, and the PAPR of each symbol is calculated independently. Unfortunately, this does not apply to the FBMC/OQAM signal, owing to the overlapping data blocks. To accurately calculate the PAPR, the overlapping between the symbol blocks must be taken into consideration.

The PAPR of pure FBMC/OQAM in $\mathrm{dB}$ can be expressed as follows:

$$
\operatorname{PAPR}(\mathrm{dB})=10 \log _{10} \frac{\max _{\mathrm{T}(\mathrm{m}-1) \leq \mathrm{t} \leq \mathrm{mT}}\left|\mathrm{s}(\mathrm{t})_{\mathrm{m}}\right|^{2}}{\mathrm{E}\left[|\mathrm{s}(\mathrm{t})|^{2}\right]}
$$

From Equation (6) the numerator represents the peak power in the duration of the $\mathrm{m}^{\text {th }}$ input data block, $\mathrm{T}(\mathrm{m}-1) \leq \mathrm{t} \leq \mathrm{mT}$, and the denominator $\mathrm{E}\left[|\mathrm{s}(\mathrm{t})|^{2}\right]$ represents the average power of the FBMC signal. 


\section{Proposed Method}

Based on previous research studies by $[17,18]$, we present our proposed PAPR reduction method, which combines DFT spreading and a modified PTS scheme. In [17], the authors proposed a technique that combines DFT spreading and a modified PTS method to satisfy the structure of visible light communication (VLC) OFDM systems.

In the proposed method, the PTS scheme is applied in the real and imaginary parts, like the method in [18] for OFDM systems. However, there is a significant difference in the creation of the candidates, given the structural differences between the FBMC/OQAM and OFDM symbols.

In [18], the complex input symbols are divided into real and imaginary parts; PTS sequences are then created at each part by multiplying the input sub-blocks with phase rotation factors. Subsequently, symbol candidates are created by combining real and imaginary part sequences. The candidates' total number is square two of the candidates in conventional PTS; therefore, the complexity is considerably higher. The proposed method reduces the PAPR of the real and imaginary parts, respectively, and the PAPR is computed by considering all the previously overlapped symbol parts. This method produces double the number of candidates, which slightly increases the computational complexity.

The next section presents the proposed method in detail.

\subsection{DFT Spreading}

The complex input data $x_{m, n}$ after passing through the FFT block are expressed as follows:

$$
X_{m, n}=C_{n, m}+j D_{n, m}
$$

The real part $C_{n, m}$ and imaginary part $D_{n, m}$ are split and multiplied by phase rotation factors, which are set such that the DFT spreading achieves the minimum PAPR value. In the proposed method, we selected the following phase factor pattern [12]:

$$
\begin{gathered}
\alpha_{n, m}=j^{n+2 m} \\
\beta_{n, m}=(-1)^{m} j(-j)^{n}
\end{gathered}
$$

Using the phase factors (8) and (9), we can satisfy a condition referred to as the identical time-shifted pattern (ITSM), which has better PAPR performance than the conventional DFT spreading method. The DFT-spread FBMC/OQAM signal equation is as follows [12]:

$$
\mathrm{s}(\mathrm{t})=\sum_{\mathrm{n}=0}^{\mathrm{N}-1} \sum_{\mathrm{m}=0}^{\mathrm{M}-1}(-1)^{\mathrm{m}}\left\{\mathrm{C}_{\mathrm{n}, \mathrm{m}} \mathrm{h}(\mathrm{t}-\mathrm{mT})+j \mathrm{D}_{\mathrm{n}, \mathrm{m}} \mathrm{h}\left(\mathrm{t}-\mathrm{mT}-\frac{\mathrm{T}}{2}\right)\right\} \mathrm{e}^{\mathrm{jn} \frac{2 \pi}{\mathrm{T}}\left(\mathrm{t}+\frac{\mathrm{T}}{4}\right)}
$$

Although ITSM-conditioned DFT spreading reduces the PAPR, it is only a marginal reduction compared to that of the DFT spreading in OFDM systems. In [12], the authors proposed a method that generates four different versions of the $\mathrm{m}^{\text {th }}$ data blocks that are identical, but with different phase shifts; further, the data block that produces the least PAPR was selected. In the proposed method, we combine a modified PTS method with ITSM-conditioned DFT spreading to achieve better PAPR reduction.

\subsection{PTS}

In the conventional PTS method, an input block is divided into V sub-blocks. Each sub-block is zero-padded to create a vector of length N. IFFT is performed separately for each sub-block, significantly increasing the computation complexity. Adjacent, interleaving, and pseudo-random are some of the widely used partitioning schemes [19]. The adjacent method is used in the proposed method, owing to its simplicity and effectiveness. From Figure 3, it can be observed that the resulting sequences are optimized by phase rotation factors $b=\left[b^{1}, b^{2}, \ldots, b^{V}\right]$, where $b^{v}=e^{j 2 \pi v / W}$ and $v=0,1, \ldots, W-1$, to create symbol candidates referred to as partial transmit sequences. This operation results in a variation of 
the peak values for the signal candidates, and the one with the minimum PAPR is chosen for transmission.

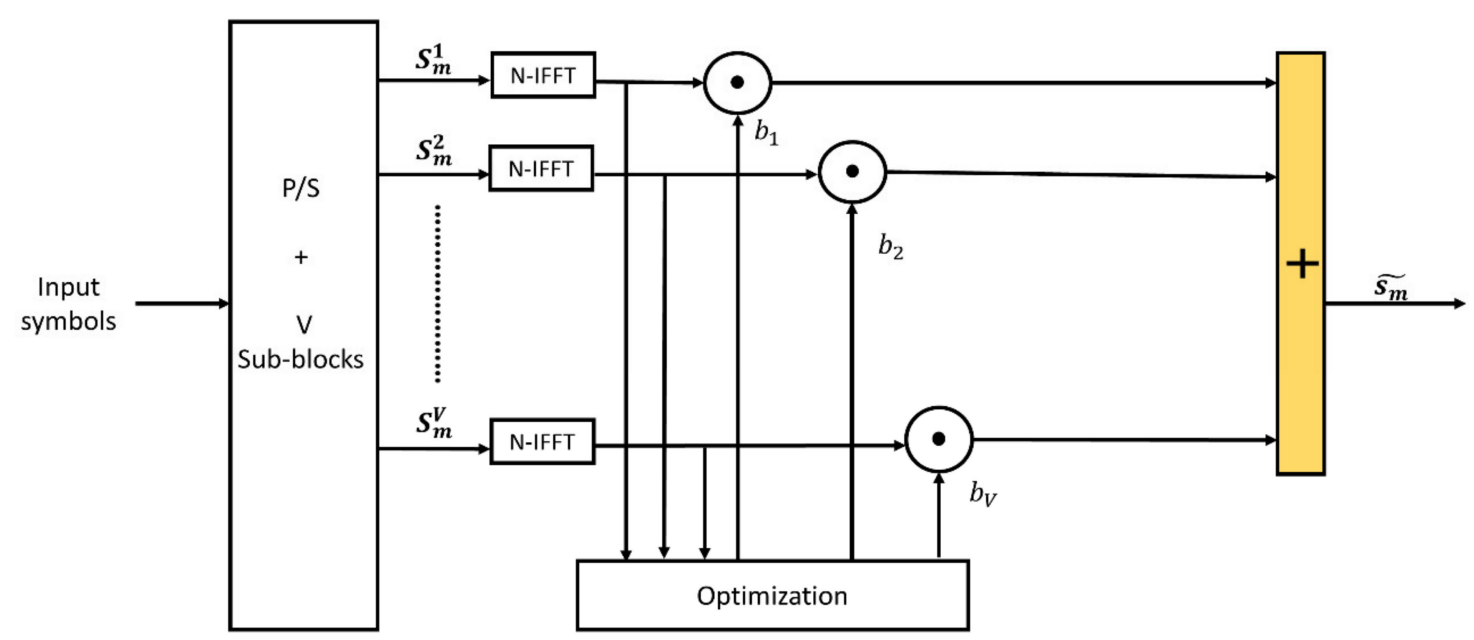

Figure 3. PTS (partial transmit sequence) scheme at the transmitter for OFDM (orthogonal frequency division multiplexing).

The total number of signal candidates depends on $\mathrm{V}$ and $\mathrm{W}$, where $\mathrm{W}$ is the number of the phase factors allowed for a single sub-block.

The process of producing the optimum phase factor vector that reduces the PAPR is as follows [6,19]:

$$
\left\{\widetilde{b^{1}}, \widetilde{b^{2}}, \ldots \widetilde{b^{V}}\right\}=\arg \min _{\left[b^{1}, \ldots, b^{v}\right]} \max _{0 \leq t \leq T}\left|\sum_{v=1}^{V} s_{m}^{v} b^{v}\right|^{2}
$$

The transmit signal after reducing the PAPR can be expressed as follows:

$$
\widetilde{\mathrm{s}_{\mathrm{m}}}(\mathrm{t})=\sum_{\mathrm{v}=1}^{\mathrm{V}} \mathrm{s}_{\mathrm{m}}^{\mathrm{v}} \widetilde{\mathrm{b}^{\mathrm{v}}}
$$

Direct application of this method to each FBMC symbol separately is not effective. As the symbols overlap, the parts where the symbols overlap will have a peak regrowth, increasing the PAPR again.

\subsection{Combination of DFT Spreading and PTS}

As shown in Figure 4, the PTS operation is performed separately at the FBMC inphase and quadrature parts. After multiplying the real and imaginary parts with the phase patterns (8) and (9), the output sequence is divided into $\mathrm{V}$ adjacent sub-blocks, which are then zero-padded to create vectors of length $\mathrm{N}$. The vectors are oversampled with $(\mathrm{L}-1) \mathrm{N}$ zeros to achieve a better PAPR representation, that mimics the continuous-time FBMC/OQAM signal. L represents the oversampling factor and it is set to $\mathrm{L} \geq 4$. The optimization process is performed consecutively for each part as follows:

1. In the simulations, we selected $W=\{-1,1\}=2$ as a phase factor because a higher value than $\mathrm{W}=2$ complicates the calculations [9]; therefore, the total number of candidates is $2^{\mathrm{V}}$.

2. After creating the PTS sequences, the output is copied $\mathrm{K}$ times and filtered with the PPN filter.

3. The PAPR of the filtered sequences is calculated, and the PAPR equations for each part are as follows:

$$
\operatorname{PAPR}_{\mathrm{r}}=\frac{\max _{(\mathrm{m}-1) \mathrm{T}+1 \leq \mathrm{t} \leq(\mathrm{k}+\mathrm{m}+1) \mathrm{T}}|\operatorname{real}(\mathrm{s}(\mathrm{t}))|^{2}}{\mathrm{E}\left[|\mathrm{s}(\mathrm{t})|^{2}\right]}
$$




$$
\operatorname{PAPR}_{\mathrm{i}}=\frac{\max _{(\mathrm{m}-1 / 2) \mathrm{T}+1 \leq \mathrm{t} \leq(\mathrm{k}+\mathrm{m}+3 / 2) \mathrm{T}}|\operatorname{imag}(\mathrm{s}(\mathrm{t}))|^{2}}{\mathrm{E}\left[|\mathrm{s}(\mathrm{t})|^{2}\right]}
$$

where PAPR ${ }_{r}$ represents the PAPR of the real part during the symbol duration $(\mathrm{m}-1) \mathrm{T}+$ $1 \leq \mathrm{t} \leq(\mathrm{k}+\mathrm{m}+1) \mathrm{T}$, and $\mathrm{PAPR}_{\mathrm{i}}$ represents the PAPR of the imaginary part with symbol duration $(\mathrm{m}-1 / 2) \mathrm{T}+1 \leq \mathrm{t} \leq(\mathrm{k}+\mathrm{m}+3 / 2) \mathrm{T}$.

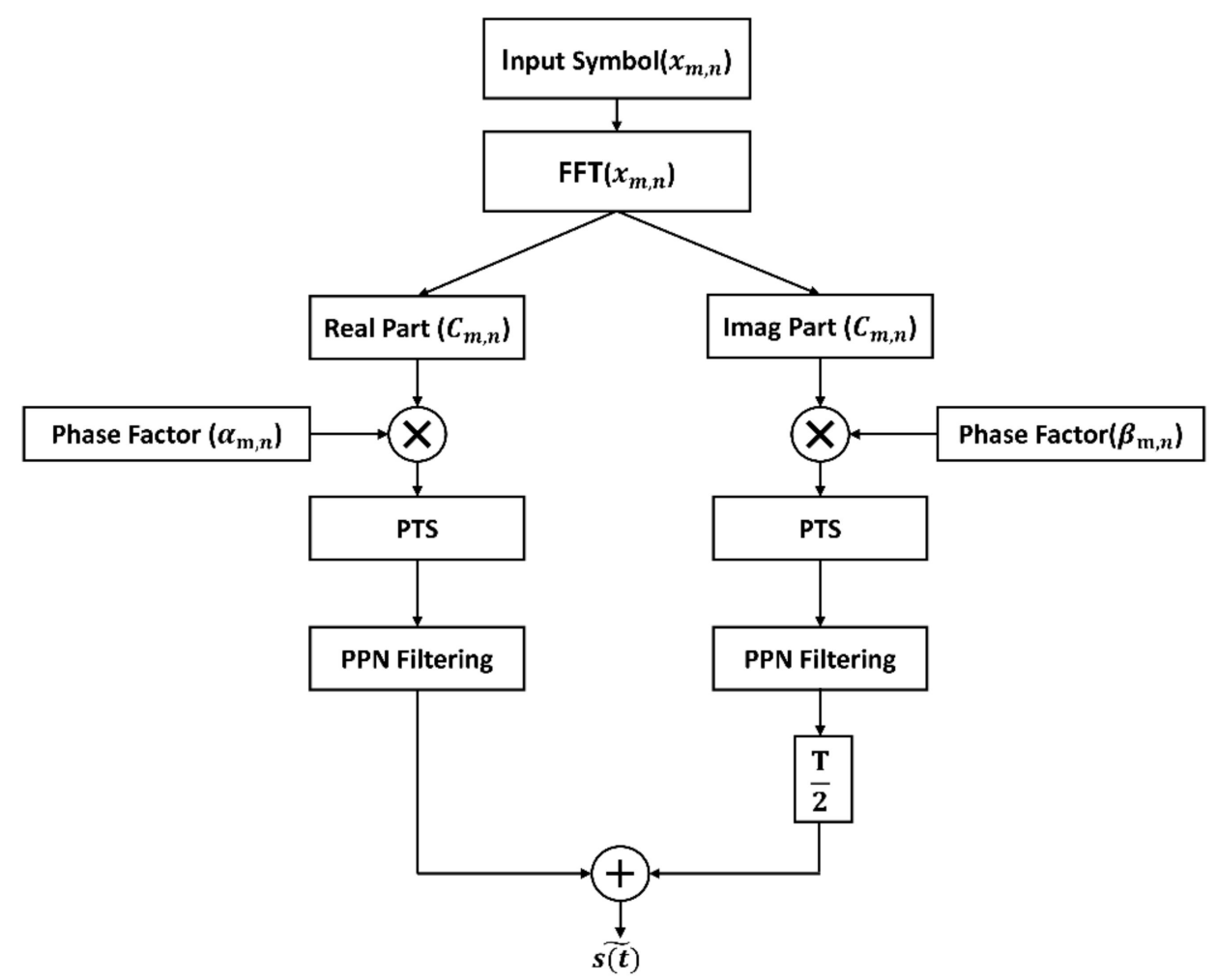

Figure 4. Block diagram of the proposed method.

It can be clearly observed from Equations (13) and (14) that while calculating the PAPR, the effect of the previous overlapped parts is also considered.

4. The selection of the optimum phase factors is as follows:

$$
\begin{gathered}
\left\{\widetilde{\mathrm{b}^{1, \mathrm{r}}}, \widetilde{\mathrm{b}^{2, \mathrm{r}}}, \ldots \widetilde{\mathrm{b}^{\mathrm{V}, \mathrm{r}}}\right\}=\arg \min _{\left[\mathrm{b}^{1}, \ldots, \mathrm{b}^{\mathrm{v}}\right](\mathrm{m}-1) \mathrm{T}+1 \leq \mathrm{t} \leq(\mathrm{k}+\mathrm{m}+1) \mathrm{T}}\left|\sum_{\mathrm{v}=1}^{\mathrm{V}} \mathrm{s}_{\mathrm{m}}^{\mathrm{v}, \mathrm{r}} \mathrm{b}^{\mathrm{v}, \mathrm{r}}\right|^{2} \\
\left\{\widetilde{\mathrm{b}^{1, \mathrm{i}}}, \widetilde{\mathrm{b}^{2, \mathrm{i}}}, \ldots \widetilde{\mathrm{b}^{\mathrm{V}, \mathrm{i}}}\right\}=\arg \min _{\left[\mathrm{b}^{1}, \ldots, \mathrm{b}^{\mathrm{v}}\right](\mathrm{m}-1 / 2) \mathrm{T}+1 \leq \mathrm{t} \leq(\mathrm{k}+\mathrm{m}+3 / 2) \mathrm{T}}\left|\sum_{\mathrm{v}=1}^{\mathrm{V}} \mathrm{s}_{\mathrm{m}}^{\mathrm{v}, \mathrm{i} \mathrm{b}^{\mathrm{v}, \mathrm{i}}}\right|^{2}
\end{gathered}
$$

5. After choosing the candidate with the least PAPR, the real and imaginary parts are combined after staggering the later by $\mathrm{T} / 2$.

\section{Simulation Results}

\subsection{PAPR Performance}

In this section, we analyze and compare the PAPR of our proposed method with other existing methods using the parameters in Table 2. Each input data block is oversampled by inserting $\mathrm{L}(\mathrm{N}-1)$ zero between QAM modulated data symbols, providing a more accurate representation of a continuous-time signal. The filter length after the oversampling is equal to $K L T$, for which $K=4$; the oversampling factor L [20] was set to 4 in all the simulations. 
Table 2. Simulation parameters for PAPR (peak-to-average power ratio) calculations.

\begin{tabular}{cc}
\hline Parameters & Value \\
\hline Modulation & 4 -QAM \\
N (IFFT/FFT size) & 64,256 \\
Filter size & 1024,4096 \\
K & 4 \\
No. of symbol blocks & 100 \\
V & 2,4 \\
W & $1,-1$ \\
L & 4 \\
\hline
\end{tabular}

Figures 5 and 6 show the PAPR CCDF plots of the pure FBMC/OQAM signal, DFT spreading with the ITSM condition, improved ITSM in [12], and our proposed method for $\mathrm{N}=64$ and $\mathrm{N}=256$. The PAPR of the pure FBMC/OQAM signal is the highest, with $10.7 \mathrm{~dB}$ and $11.3 \mathrm{~dB}$ at a probability of $10^{-3}$, respectively. For the sub-carrier value $\mathrm{N}$ $=64$, applying DFT spreading to pure FBMC/OQAM, while satisfying the ITSM phase condition, reduces the PAPR by approximately $1.6 \mathrm{~dB}$, and combining it with the modified PTS leads to a reduction of approximately $3.4 \mathrm{~dB}$ and $4 \mathrm{~dB}$ for the sub-block values, $\mathrm{V}=2$ and $\mathrm{V}=4$, respectively. Similarly, for $\mathrm{N}=256$, the PAPR reduction is approximately $1.8 \mathrm{~dB}$ for DFT spreading, $3.2 \mathrm{~dB}$, and $3.8 \mathrm{~dB}$ for $\mathrm{V}=2$ and $\mathrm{V}=4$, respectively. The proposed method exhibits better PAPR performance than the improved ITSM [12], with a reduction of approximately $0.4 \mathrm{~dB}$ and $0.3 \mathrm{~dB}$. For small sub-block values such as $\mathrm{V}=2$ and $\mathrm{V}=4$, the results show an acceptable reduction in PAPR. As seen from Figures 5 and 6, the PAPR reduces significantly when $\mathrm{V}=4$, with a considerable increase in the computation complexity. For higher sub-block values, the computation complexity increases exponentially. Thus, the implementation becomes more difficult. Increasing the order of QAM symbols will lead to an increase in the PAPR in the presence of the proposed method with average percentage of approximately $6 \%$ at probability $10^{-3}$.

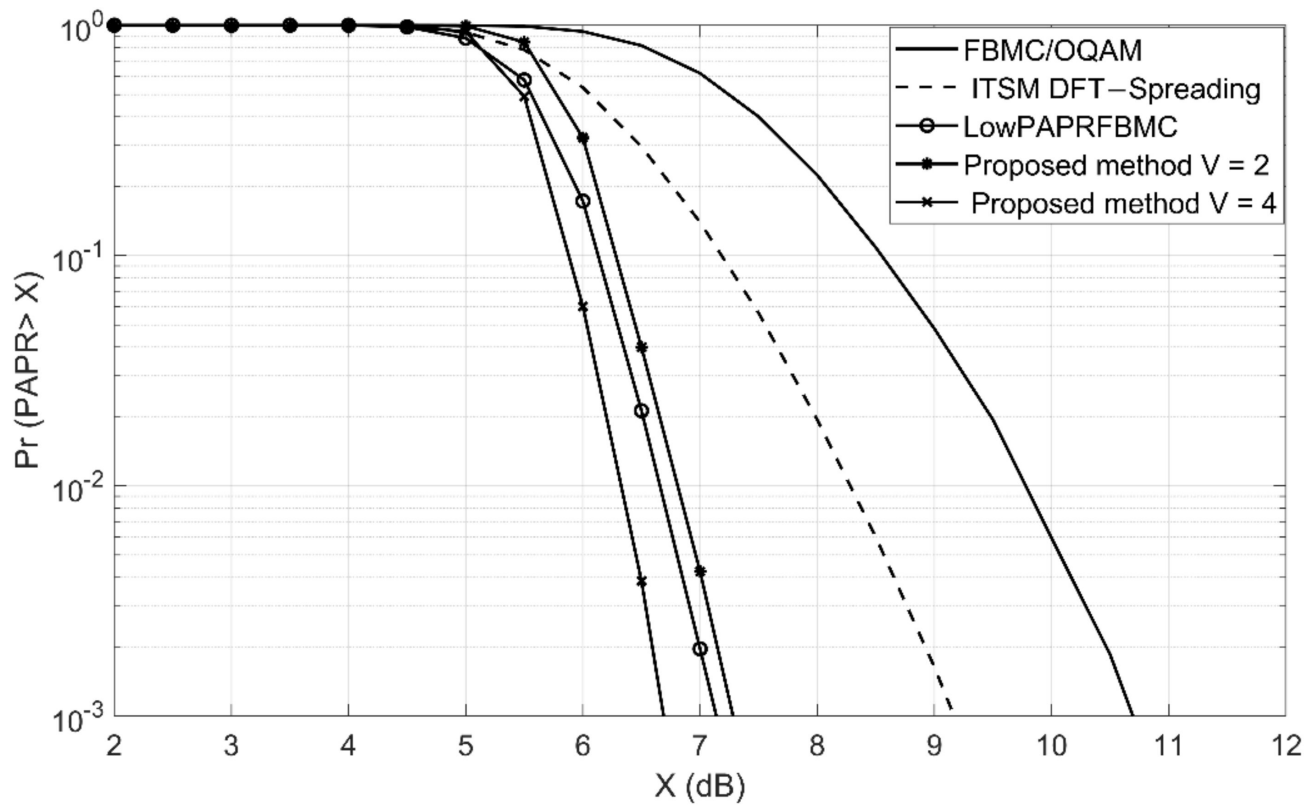

Figure 5. PAPR (peak-to-average power ratio) comparison of several reduction methods for $\mathrm{N}=64$. 


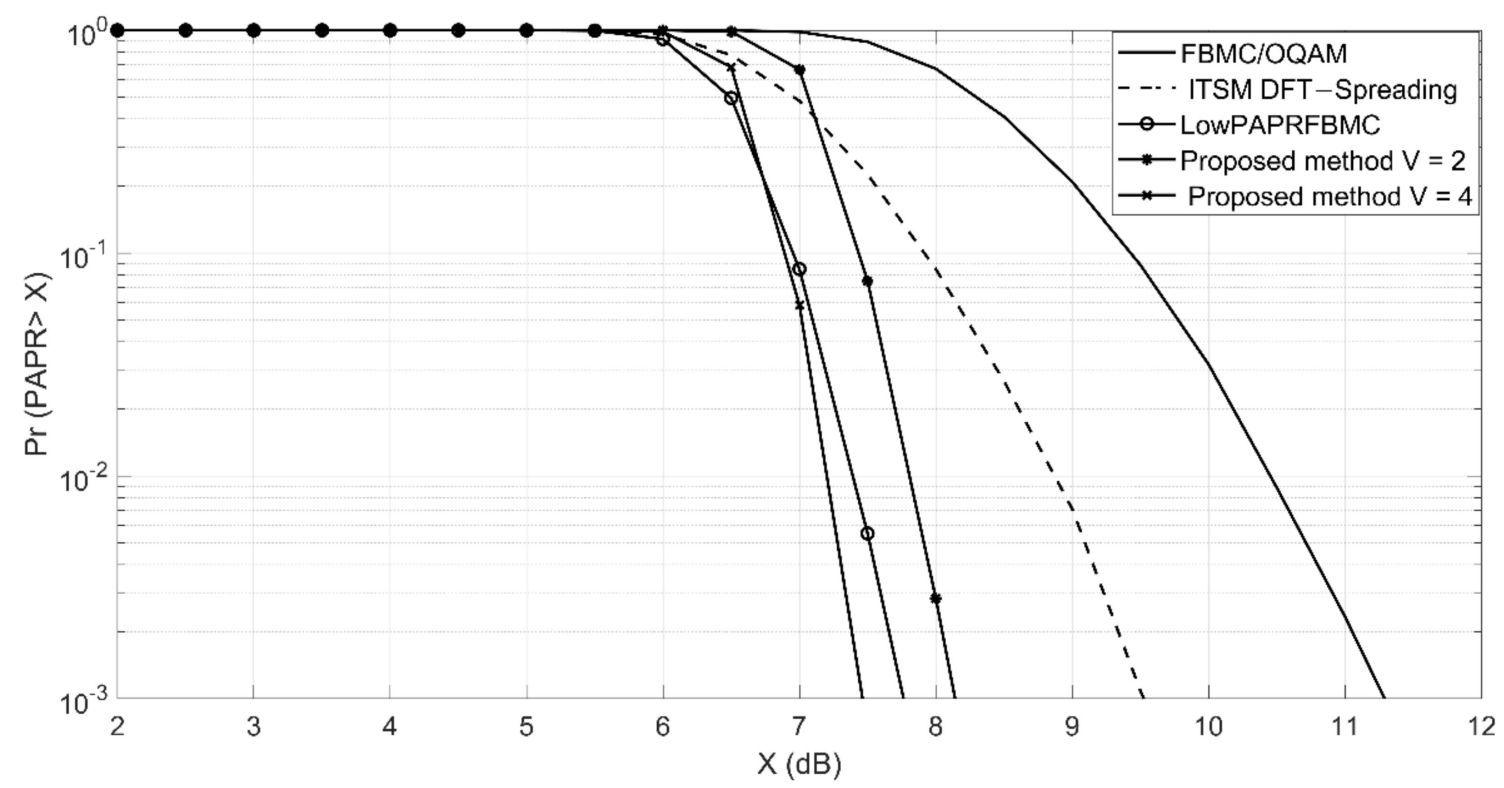

Figure 6. PAPR (peak-to-average power ratio) comparison of several reduction methods for $\mathrm{N}=256$.

From the results, we conclude that the proposed method outperforms the other mentioned methods with a reduction percentage of $29 \%$ and $36.5 \%$ for the sub-carrier value of $\mathrm{N}=64$, and $27.4 \%$ and $33.6 \%$ for the sub-carrier value of $\mathrm{N}=256$.

\subsection{Computational Complexity}

One of the main concerns when designing a PAPR reduction method is its effect on the performance of the multicarrier systems. PAPR reduction methods are known to increase the system's computational complexity; therefore, implementation becomes even more challenging. This section analyzes the computation complexity of the proposed method and compares it with those of the pure FBMC/OQAM signal, DFT spreading FBMC/OQAM, and modified PTS FBMC/OQAM. The computation complexity calculations mainly involve the total number of real multiplications (RMs) and real additions. For our analysis, we focused on the RMs at the transmitter side only, including IDFT operation, DFT spreading operation, PPN filtering, and phase factor optimization.

As mentioned in the previous section, for the IDFT operation, LN IFFT points were performed. The number of multiplications related to the IDFT operation for complex input data equals $\frac{L N}{2} \log _{2} N+L N / 2$ [12]. Multiplying two complex numbers results in four RMs and three additions; therefore, the total number of multiplications are $4 \times$ $\left[\frac{L N}{2} \log _{2} N+L N / 2\right]$ [12]. The total number of IDFTs for the PTS operation is $2 \mathrm{~V}$ because the partitioning is separately performed in the real and imaginary parts; therefore, the total number of multiplications is $8 \mathrm{~V} \times\left[\frac{L N}{2} \log _{2} N+L N / 2\right]$ for the proposed method. For DFT spreading, the total number of multiplications is $4 \times\left[\mathrm{N} / 2 \log _{2} N\right]$. The PPN filtering in the proposed method is performed $2^{\mathrm{V}} \times 8 \mathrm{KN}$ times for the real and imaginary parts. Finally, the phase factor searching for the proposed method was set to $8 \times\left[2^{v-1} N(V+1)\right][19]$.

Table 3 summarizes the main calculations and comparison between pure FBMC/OQAM, DFT-FBMC/OQAM, PTS FBMC/OQAM, and the proposed method. The simulation parameters are $\mathrm{N}=64, \mathrm{~L}=4, \mathrm{~K}=4, \mathrm{~W}=2\{1,-1\}, \mathrm{V}=4$. As presented in the table, the total number of RMs of the pure FBMC/OQAM is the least, whereas those of the proposed method are the highest. As mentioned previously, most PAPR reduction methods work well in reducing the PAPR. However, it comes with the cost of increasing the computation complexity, BER, and spectral efficiency degradation. The conventional PTS method is known for its high computational complexity, mainly when performing V-IFFT operations and phase searching. As the number of sub-blocks increases, the computational complexity 
increases significantly. In [21], the author proposed reducing the PAPR of OFDM/OQAM by dividing the FBMC signal into segments and applying the PTS reduction scheme on each segment separately. Although the computational complexity of this method is less than that of the original PTS method, the structure of the OFDM/OQAM is different; thus, it cannot be directly applied to FBMC/OQAM. In the proposed method, as mentioned earlier, all previous operations are performed for the real and imaginary parts; therefore, we conclude that the proposed method has the highest computational complexity.

Table 3. Simulation parameters for computation complexity.

\begin{tabular}{ccc}
\hline Method & $\begin{array}{c}\text { Calculations } \\
\text { (per 1 Symbol Period) }\end{array}$ & $\begin{array}{c}\text { No. of Real } \\
\text { Multiplications }\end{array}$ \\
\hline FBMC/OQAM & 2 IDFTs, 2 PPNs & 12,288 \\
\hline DFT-FBMC/OQAM & 1 DFT, 2 IDFTs, 2 PPNs & 13,184 \\
\hline PTS FBMC/OQAM & $\begin{array}{c}\text { 2V IDFTs, } 2 \times 2^{\mathrm{V}} \text { PPN, phase } \\
\text { factor searching } 2^{\mathrm{V}} \mathrm{N}(\mathrm{V}+1)\end{array}$ & 86,016 for $\mathrm{V}=4$ \\
\hline Proposed method & $\begin{array}{c}\text { 1 DFT, 2V IDFTs, } 2 \times 2 \mathrm{~V} \text { PPN, } \\
\text { phase factor searching 2V } \\
\text { N(V+1) }\end{array}$ & 86,912 for $\mathrm{V}=4$ \\
\hline
\end{tabular}

\subsection{BER Performance}

BER is an important measure to evaluate the effect of the PAPR reduction method on the transmitted data. Many PAPR reduction methods successfully reduce the PAPR. However, they may distort the original signal, which may affect the BER of the system. We evaluated the proposed method's performance by adding the effect of the nonlinear HPA distortion to the transmitted signal. The HPA model used in our simulation is a soft envelope limiter (SEL) [22]. The SEL amplifier affects only the amplitude of the input signal, whereas the phase remains unchanged. After passing through the SEL model, the transmitted signal is as follows:

$$
\widetilde{\mathrm{x}}(\mathrm{t})=\left\{\begin{array}{l}
x(\mathrm{t}), \quad|\mathrm{x}(\mathrm{t})| \leq \mathrm{A}_{\text {sat }} \\
\mathrm{A}_{\text {sat }} \mathrm{e}^{\mathrm{j} \varphi(\mathrm{t})},|\mathrm{x}(\mathrm{t})|>\mathrm{A}_{\text {sat }}
\end{array}\right.
$$

where $\varphi(t)$ represents the signal phase, and $A_{\text {sat }}$ is the saturation or clipping level of the HPA. One of the critical parameters in HPAs, referred to as the input back-off (IBO) power, determines the maximum power of the HPA before it moves to the saturation region. As the input signal's amplitude increases, it causes the HPA to operate in the saturation region or eventually in its nonlinear region, which causes distortion of the transmitted signal. The $\mathrm{IBO}$ in $\mathrm{dB}$ is defined as follows [23]:

$$
\mathrm{IBO}=10 \log _{10}\left(\frac{\mathrm{A}_{\mathrm{sat}}{ }^{2}}{\sigma^{2}}\right)
$$

The variance of the input signal is denoted as $\sigma^{2}$. Table 4 lists the parameters used in the simulation. The BER of the pure FBMC/OQAM without the HPA effect, with the HPA effect, and the proposed method with the HPA effect for different IBO values are presented. Figures 7 and 8 show the BER plot with 256 sub-carriers and IBO values of $7 \mathrm{~dB}$ and $3 \mathrm{~dB}$, respectively. As shown in the graphs, the BER of the pure FBMC/OQAM increases as the IBO value decreases when the HPA effect is added. At probabilities of $10^{-2}$, the BER value is above $8 \mathrm{~dB}$, and with the addition of the HPA effect, the BER is above $10 \mathrm{~dB}$ and $18 \mathrm{~dB}$ for IBO values of $7 \mathrm{~dB}$ and $3 \mathrm{~dB}$, respectively. When the proposed method is applied, the BER is reduced by $1.2 \mathrm{~dB}$ and $6.7 \mathrm{~dB}$, achieving a $13 \%$ and $37 \%$ improvement in the BER performance, respectively. The results indicate that the proposed method helps to mitigate the clipping effect of the SEL HPA by reducing the peaks of the FBMC/OQAM. 
Table 4. Simulation parameters for BER (bit-error-rate) calculations.

\begin{tabular}{cc}
\hline Parameters & Value \\
\hline Modulation & $4-\mathrm{QAM}$ \\
N (IFFT/FFT size) & 256 \\
Filter size & 4096 \\
K & 4 \\
No. of symbol blocks & 100 \\
V & 4 \\
W & $1,-1$ \\
L & 4 \\
IBO & $7 \mathrm{~dB}, 3 \mathrm{~dB}$ \\
\hline
\end{tabular}

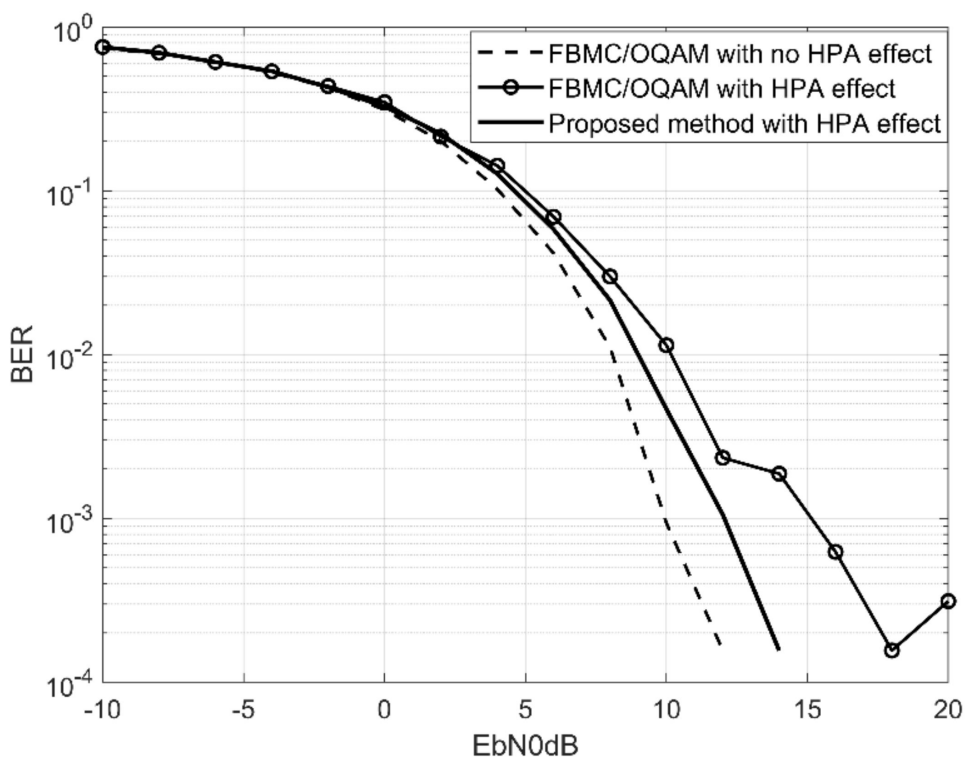

Figure 7. BER (bit-error-rate) comparison with HPA (high-power amplifier) effect for IBO $=7 \mathrm{~dB}$ (input back-off).

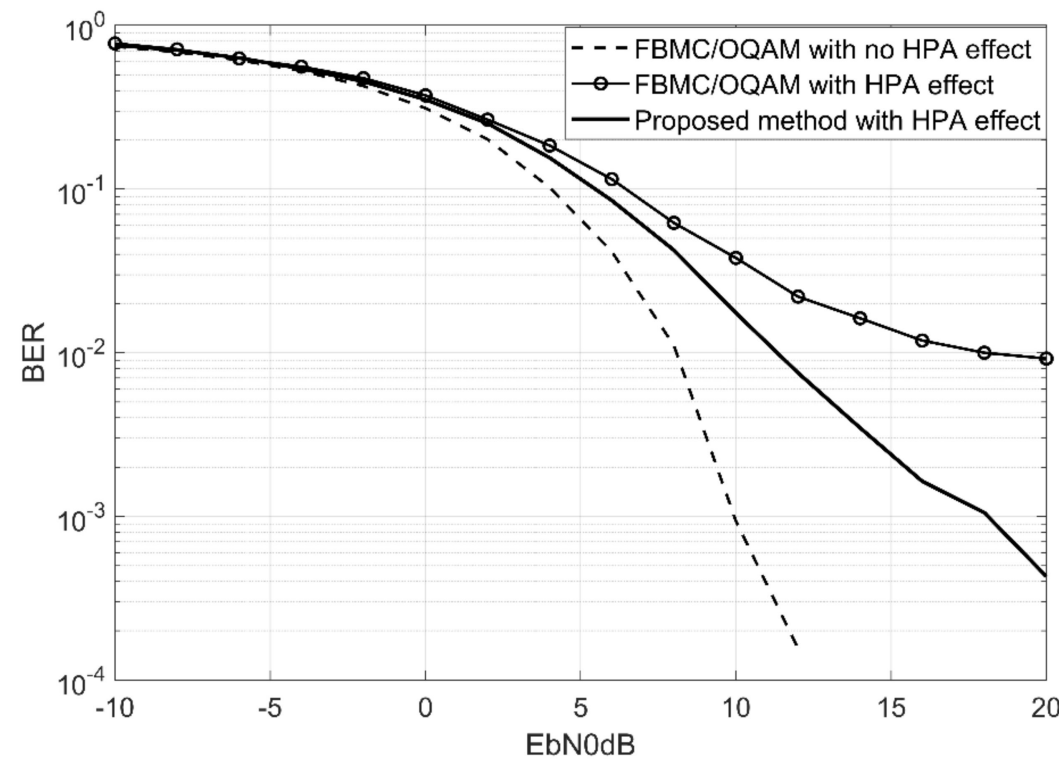

Figure 8. BER (bit-error-rate) comparison with HPA (high-power amplifier) effect for IBO $=3 \mathrm{~dB}$ (input back-off). 


\section{Conclusions}

The proposed method introduces a new approach to reducing the PAPR by combining DFT spreading with a modified PTS method. After conducting several simulations, the results showed a significant reduction in the PAPR with an average of $32.8 \%$, without worsening the BER performance. The computation complexity analysis showed that the proposed method requires considerably more computation power than the previous methods, mainly from simultaneously performing V-IFFTs, phase searching, and candidate selection at both the real and imaginary parts. Additionally, the BER performance of the proposed method with the addition of the HPA effect shows an improvement with a $25 \%$ reduction in the BER when the HPA effect is severe. The efficiency of our method could be further improved by reducing the computational complexity and eliminating redundant SI bits.

Author Contributions: Conceptualization, N.A.H. and S.C.; methodology, N.A.H.; software, N.A.H. and Z.Z.; validation, D.K., Z.Z. and N.A.H.; formal analysis, N.A.H.; investigation, N.A.H.; resources, N.A.H. and Z.Z.; data curation, N.A.H.; writing-original draft preparation, N.A.H.; writingreview and editing, N.A.H. and S.C.; visualization, N.A.H., Z.Z. and D.K.; supervision, S.C.; project administration, S.C.; funding acquisition, S.C. All authors have read and agreed to the published version of the manuscript.

Funding: This research received no external funding.

Acknowledgments: This research was supported by the Commercialization Promotion Agency for R\&D Outcomes (COMPA) funded by the Ministry of Science and ICT (MSIT) (2020K000081).

Conflicts of Interest: The authors declare no conflict of interest.

\section{References}

1. Farhang-Boroujeny, B. Filter bank multicarrier modulation: A waveform candidate for 5G and beyond. Adv. Electr. Eng. 2014, 1-25. [CrossRef]

2. Banelli, P.; Buzzi, S.; Colavolpe, G.; Modenini, A.; Rusek, F.; Ugolini, A. Modulation Formats and Waveforms for 5G Networks: Who Will Be the Heir of OFDM? An overview of alternative modulation schemes for improved spectral efficiency. IEEE Signal Process. Mag. 2014, 31, 80-93. [CrossRef]

3. Jiang, W.; Schellmann, M. Suppressing the out-of-band power radiation in multi-carrier systems: A comparative study. In Proceedings of the 2012 IEEE Global Communications Conference (GLOBECOM), Anaheim, CA, USA, 3-7 December 2012; pp. 1477-1482. [CrossRef]

4. Han, S.H.; Lee, J.H. An overview of peak-to-average power ratio reduction techniques for multicarrier transmission. IEEE Wirel. Commun. 2005, 12, 56-65. [CrossRef]

5. Lu, S.; Qu, D.; He, Y. Sliding Window Tone Reservation Technique for the Peak-to-Average Power Ratio Reduction of FBMCOQAM Signals. IEEE Wirel. Commun. Lett. 2012, 1, 268-271. [CrossRef]

6. Wang, H.; Wang, X.; Xu, L.; Du, W. Hybrid PAPR Reduction Scheme for FBMC/OQAM Systems Based on Multi Data Block PTS and TR Methods. IEEE Access 2016, 4, 4761-4768. [CrossRef]

7. He, Z.; Zhou, L.; Chen, Y.; Ling, X. Low-Complexity PTS Scheme for PAPR Reduction in FBMC-OQAM Systems. IEEE Commun. Lett. 2018, 22, 2322-2325. [CrossRef]

8. Hanprasitkum, A.; Numsomran, A.; Boonsrimuang, P.; Boonsrimuang, P. Improved PTS method with new weighting factor technique for FBMC-OQAM systems. In Proceedings of the 2017 19th International Conference on Advanced Communications Technology (ICACT), Bongpyeong, Korea, 19-22 February 2017. [CrossRef]

9. Moon, J.; Nam, Y.; Kim, J. PAPR Reduction in the FBMC-OQAM System via Segment-Based Optimization. IEEE Access 2018, 6, 4994-5002. [CrossRef]

10. Ren, S.; Deng, H.; Qian, X.; Liu, Y. Sparse PTS scheme based on TR schemes for PAPR reduction in FBMC-OQAM systems. IET Commun. 2018, 12, 1722-1727. [CrossRef]

11. Qu, D.; Lu, S.; Jiang, T. Multi-Block Joint Optimization for the Peak-to-Average Power Ratio Reduction of FBMC-OQAM Signals. IEEE Trans. Signal Process. 2013, 1605-1613. [CrossRef]

12. Na, D.; Choi, K. Low PAPR FBMC. IEEE Trans. Wirel. Commun. 2017, 17, 182-193. [CrossRef]

13. Aboul-Dahab, M.A.; Foud, M.M.; Roshdy, R.A. Generalized Discrete Fourier Transformfor FBMC Peak to Average Power Ratio Reduction. IEEE Access 2019, 7, 81730-81740. [CrossRef]

14. Nissel, R.; Rupp, M. Pruned DFT-Spread FBMC: Low PAPR, Low Latency, High Spectral Efficiency. IEEE Trans. Commun. 2018, 66, 4811-4825. [CrossRef] 
15. Yuen, C.H.; Amini, P.; Farhang-Boroujeny, B. Single carrier frequency division multiple access (SC-FDMA) for filter bank multicarrier communication systems. In Proceedings of the 5th IEEE International Conference on Cognitive Radio Oriented Wireless Networks and Communications (CROWNCOM), Cannes, France, 9-11 June 2010; pp. 1-5.

16. Bellanger, M.D.; LeRuyet, D.; Roviras, D.; Terre, M.; Nossek, J.; Baltar, L.; Bai, Q.; Waldhauser, D.; Renfors, M.; Ihalainen, T.; et al. FBMC Physical Layer: A Primer; PHYDYAS, 2010; Available online: http:/ / www.ict-phydyas.org/ (accessed on 5 March 2021).

17. Fulai, Z.; Luokun, L.; Jinjin, Y. DFT-spread combined with PTS method to reduce the PAPR in VLC-OFDM system. In Proceedings of the 2014 IEEE 5th International Conference on Software Engineering and Services Science, Beijing, China, 27-29 June 2014; pp. 629-632. [CrossRef]

18. Wu, X.; Mao, Z.; Wang, J.; Zhou, B. A Novel PTS Technique with Combinative Optimization in Real Part and Imaginary Part for PAPR Reduction in OFDM Systems. In Proceedings of the 2009 Third International Conference on Next Generation Mobile Applications, Services and Technologies, Cardiff, Wales, UK, 16-19 September 2009; pp. 215-218. [CrossRef]

19. Jawhar, Y.A.; Audah, L.; Taher, M.A.; Ramli, K.N.; Shah, N.S.M.; Musa, M.; Ahmed, M.S. A Review of Partial Transmit Sequence for PAPR Reduction in the OFDM Systems. IEEE Access 2019, 7, 18021-18041. [CrossRef]

20. Wang, C.-L.; Ouyang, Y. Low-complexity selected mapping schemes for peak-to-average power ratio reduction in OFDM systems. IEEE Trans. Signal Process. 2005, 53, 4652-4660. [CrossRef]

21. Ye, C.; Li, Z.; Jiang, T.; Ni, C.; Qi, Q. PAPR Reduction of OQAM-OFDM Signals Using Segmental PTS Scheme With Low Complexity. IEEE Trans. Broadcast. 2014, 60, 141-147. [CrossRef]

22. Vihriala, J.; Ermolova, N.; Lahetkangas, E.; Tirkkonen, O.; Pajukoski, K. On the Waveforms for 5G Mobile Broadband Communications. In Proceedings of the 2015 IEEE 81st Vehicular Technology Conference (VTC Spring), Glasgow, UK, 11-14 May 2015; pp. 1-5. [CrossRef]

23. Bouhadda, H.; Shaiek, H.; Roviras, D.; Zayani, R.; Medjahdi, Y.; Bouallegue, R. Theoretical analysis of BER performance of nonlinearly amplified FBMC/OQAM and OFDM signals. EURASIP J. Adv. Signal Process. 2014. [CrossRef] 